\title{
Recurrent Childhood Undifferentiated High Grade Pleomorphic Sarcoma of Bone
}

National Cancer Institute

\section{Source}

National Cancer Institute. Recurrent Childhood Undifferentiated High Grade Pleomorphic Sarcoma of Bone. NCI Thesaurus. Code C115368.

The reemergence of undifferentiated high grade pleomorphic sarcoma of bone in childhood after a period of remission. 